\title{
Manuel Ghilarducci*
}

\section{Die Funktion der Tropen in Aleksandr Prochanovs literarischer Publizistik}

\author{
Eine dekonstruktivistische Annäherung
}

DOI 10.1515/slaw-2017-0007

Summary: This paper investigates the tropological structure of Aleksandr Prochanov's journalistic writing from a deconstructive perspective. The aim of my analysis is to show that the presence of tropes in Prochanov's journalism cannot simply be explained as a political-ideological manipulation of literary language, as stated by most of the scholars. In my opinion it is connected to the rhetorical nature of language, i. e. to the possibility to speak and write in images and thus to subvert the relation between signified and signifier. As I show in the analysis of the exemplary text Stalin is not bronze but the speed of light, the tropological structure of the language deconstructs not only the text, but the relationship between the author and the text and thus leads us to a reinterpretation of the literary journalism as such.

Keywords: Prochanov, Deconstruction, Rhetoric, Politics, Tropes

\section{Einleitung}

Verfügt eine Schriftstellerin bzw. ein Schriftsteller über genug politisches Kapital im literarischen Feld, so kann sie bzw. er sich in belletristischen und publizistischen Schriften zu öffentlichen Themen äußern. In dem weit bekannten Phänomen der literarischen Publizistik verschwimmen zwei Grenzen: Die zwischen dem Literarischen und dem Politischen, wie Pierre Bourdieus Kultursoziologie gezeigt hat (vgl. Bourdieu 1992: 298-371) und die zwischen Belletristik und Publizistik oder mit anderen Worten zwischen dem Literarischen und dem Publizistischen.

Die Wechselbeziehung zwischen dem Literarischen und dem Publizistischen stellt meines Erachtens kein Hindernis zu einer literaturwissenschaftlichen Auseinandersetzung mit solchen ,hybriden“ Texten dar. Gerade diese Hybridität liefert die Chance zu einer neuen und produktiven Interpretation der Rolle der

*Kontaktperson: M. A. Manuel Ghilarducci, Humboldt-Universität zu Berlin, Institut für Slawistik, Dorotheenstraße 65, 10117 Berlin, E-Mail: manuel.ghilarducci@hu-berlin.de 
Sprache in der literarischen Publizistik und, daran anschließend, zu einigen Bemerkungen über die rhetorische Natur der Sprache überhaupt. Der Versuch einer eindeutigen Gattungszuordnung des Textes soll demnach vermieden und die Aufmerksamkeit auf das textuelle Verfahren an sich gerichtet werden. Dieses kann nicht nur als eine Manipulierung des figurativen Sprachgebrauchs zu politisch-ideologischen Zwecken betrachtet werden.

Zwar ermöglicht die dynamische Form der literarischen Publizistik der Autorin bzw. dem Autor, mit literarischen Techniken und politischen Diskursen zu spielen und somit auf eine originelle Art und Weise an der öffentlichen Kommunikation zu partizipieren (vgl. Averbeck-Lietz 2013: 285). Es lässt sich allerdings infrage stellen, dass das Literarische in der Publizistik nur als Mittel zum Zweck zu verstehen ist. Die Verwendung von Tropen in einem stark ideologisierten bzw. politisierten Text kann zweifelsohne zu einer Intensivierung der Appellfunktion führen. An einer totalen Kontrolle der Rhetorizität der literarischen Sprache seitens der Autorin bzw. des Autors lässt sich dennoch zweifeln.

Die literarische Publizistik zeigt, dass die Dichotomie literarisch/nicht literarisch nicht immer fundiert ist und gerade durch die Verwendung figurativer Sprache in solchen Texten sich selbst dekonstruiert. In einem literarisch publizistischen Text vollziehen sich eine Konstruktion und eine Dekonstruktion zugleich. Zum einen werden ideologische und politische Konzepte sprachlich konstruiert und verbildlicht. Zum anderen verweisen aber diese sprachlichen Bilder auf sich selbst sowie auf das textuelle bzw. rhetorische Verfahren zurück. Indem sie Signifikanten und Signifikate voneinander lösen, wirken sie als ein rhetorisches Spiel mit der Sprache und bleiben selbstreferenziell. Der Text agiert somit nahezu autonom: Er kann nicht mehr als das bloße Produkt einer Autorinstanz betrachtet werden, sondern er dekonstruiert sich selbst und auch die Autorinstanz, die somit als eine leere Trope erscheint.

Wie dieses komplexe Phänomen geschehen kann, wird in diesem Beitrag am Beispiel der Publizistik Aleksandr Prochanovs gezeigt. Aleksandr Prochanov ( $\left.{ }^{\star} 1938\right)$ ist im heutigen literarischen Feld Russlands wegen seiner politischen Tätigkeit und seiner literarischen und publizistischen Texte eine bekannte Persönlichkeit ${ }^{1}$. Prochanov vertritt eine imperialistische, nationalistische, patriarchalische und restaurative Ideologie ${ }^{2}$, die sowohl seine Belletristik als auch seine

1 Die zunehmende Anerkennung Prochanovs im literarischen Feld nach der Verleihung von Literaturpreisen (also drei Jahre nach dem Nacional'nyj bestseller) wurde marktstrategisch genutzt, um auch seine Publizistik auf dem Buchmarkt zu fördern. 2009 bekam Prochanov auch den Bunin-Preis (Buninskaja premija).

2 Prochanov vertritt zum Teil auch eurasistische Vorstellungen. Der Eurasismus kann als eine Ideologie bezeichnet werden, die mittels Synekdochen, Metaphern operiert und somit tropolo- 
Publizistik prägt und zum Teil als nationalbolschewistisch bezeichnet werden $\mathrm{kann}^{3}$.

Es ist unumstritten, dass Prochanovs Texte politische Ziele verfolgen. In seiner Publizistik kommen Themen und Motive zum Ausdruck, die auch seine Prosa prägen: Kriegsereignisse (Čečenskij bljuz 'Tschetschenischer Blues', 2002), Machtintrigen (Gospodin Geksogen 'Herr Hexogen', 2002, und Poslednij soldat imperii 'Der letzte Soldat des Imperiums', 2003) und starke, männliche Heldenfiguren $^{4}$ (Russkij 'Der Russe', 2011). Die genannten Themen und Bilder entsprechen einem dichotomischen Denken: Binäre Oppositionen wie Russland-Europa (bzw. USA), svoё-čužoe ,Eigenes-Fremdes', Kolonisierende-Kolonisierte und Vergangenheit-Gegenwart bilden den Kern der Texte Prochanovs 5 .

In Prochanovs ganzem Werk kommen, wie Alexandra Mey, Sergej Beljakov und Elizabeth Rich gezeigt haben, immer gleiche thematische und formelle Kon-

gisch konstruiert wird: „Zwischen Slavophilie und Westlertum, zwischen Europa und Asien bezeichnet Eurasismus den Versuch, durch Abgrenzung von Anderen und Konstruktion des Eigenen zu einer ganzheitlichen Identität zu gelangen. Das Konstitutivum der Identität ist im eurasischen Verständnis der mehrfach semantisierte Raum“ (Klitsche-Sowitzki 2011: 144).

3 Auf die Geschichte der nationalbolschewistischen Bewegung kann ich hier aus Platzgründen nicht ausführlich eingehen. Die Nationalbolschewiste Partei, die 1993 gegründet und 2007 als extremistische Organisation verboten wurde, präsentiert sich als eine populistische Alternative sowohl zu den rechten als auch zu den linken Parteien: „Мы не правые и не левые, мы - НБП!“ [,Wir sind keine Linken und auch keine Rechten - wir sind die NBP!“] (http://nbp-rostov.front.ru/ ideology_noleftnorait.htm. Letzter Zugriff: 29.07.2015). Diese Losung stammt von Zahar Prilepin, der wie Prochanov Publizist und Schriftsteller ist. Dazu s. Fußnote 9.

4 Der ,authentische' russische Nationalheld soll für Prochanov einen starken Charakter, eine absolute moralische Integrität und Kriegserfahrungen haben. Prochanov mythisiert den Krieg: Im Krieg könne sich der russische Mann, der von der Geburt an über ,außerordentliche‘ geistige und körperliche Fähigkeiten verfügt, noch mehr perfektionieren und nahezu den Status eines ,Übermenschen' erlangen. Es erübrigt sich, zu sagen, dass Prochanov eine absolut patriarchaliche Weltvorstellung hat, in der Frauen nur als Stütze für den Mann (als Mütter und Ehefrauen) ihren Platz finden. Eine ausführliche Studie der Männlichkeits- und Weiblichkeitsbilder bei Prochanov aus dekonstruktivistischer bzw. genderkritischer Perspektive ist meines Erachtens wünschenswert, denn die Mythisierung des Krieges und patriarchalische Auffassungen sind kein isoliertes Phänomen bei Prochanov. Sie sind auch bei anderen Schriftsteller-Nationalbolschewisten (wie Eduard Limonov oder Zahar Prilepin) und im postsowjetischen Film zu finden: Man denke nur an Aleksej Balabanovs Filme Brat 'Der Bruder', Brat 2 'Der Bruder 2' (Danila Bagrov) und Vojna 'Der Krieg' (Ivan Ermakov). Mark Lipoveckij hat in Paralogii die Besonderheiten dieser Ästhetik, die Themen und Bilder des Sozialistischen Realismus mit postmodernen Techniken verbindet, ausführlich untersucht (vgl. Lipoveckij 2008: 720-755).

5 Selbstverständlich handelt es sich hier um semiotisierte Oppositionen, die immer nach demselben Muster funktionieren: Einer der Pole ist immer positiv und der entgegengesetzte immer negativ besetzt. 
stellationen vor (vgl.: Mey 2004: 230, 253; Beljakov 2009; Rich 1995: 23-24). Insbesondere Beljakov spricht zu Recht von einer ,seriellen Prosa' (vgl. Beljakov 2009) und deutet somit implizit auf ein automatisiertes und sich wiederholendes textuelles Verfahren hin. Die Automatisierung des Schreibverfahrens ist meines Erachtens ein zentrales Element bei Prochanov, das relevante Konsequenzen auf der Ebene sowohl der Autorschaft als auch der Textur hat. Aufgrund von diesem Aspekt lässt sich das komplexe Spannungsfeld zwischen dem Politischen, dem Publizistischen und dem Literarischen bei Prochanov nicht auf eine bloße Instrumentalisierung der Literatur reduzieren.

Wie dieses Spannungsfeld sich konzeptualisieren lässt und wie die tropologische Struktur der literarischen Publizistik Prochanovs zu schildern ist, soll in diesem Beitrag verdeutlicht werden.

\section{Die (Auto-)Dekonstruktion der Publizistik Prochanovs}

Die Wechselwirkung zwischen Belletristik und Publizistik bei Prochanov manifestiert sich bereits prima facie: Seine Romane enthalten publizistische Elemente und seine Publizistik weist eine deutliche poetologische Struktur auf. Die Funktion und die Struktur des Publizistischen in Prochanovs Belletristik wurden am ausführlichsten von Aleksandra Mey und Marina Erofeeva untersucht (vgl. Mey \& Erofeeva 2007: 179-183 und Erofeeva 2009: 50-52). Dies geschah unter der Annahme, dass Literatur politisiert und somit zu einem Instrument der Verbreitung von Ideologien werden kann. Eine Analyse des Literarischen sowie der Rhetorizität in Prochanovs Publizistik liegt allerdings nach meinem Wissen noch nicht vor. Dieses Ziel verfolgt der vorliegende Beitrag.

Um die Wechselbeziehung zwischen dem Politischen, dem Publizistischen und dem Literarischen bei Prochanov zu erhellen, erscheint mir als sinnvoll, die Funktion der tropologischen Struktur in seiner Publizistik aus dekonstruktivistischer Perspektive zu untersuchen. Somit vollzieht sich ein Perspektivenwechsel im Vergleich zu den Beiträgen von Mey und Erofeeva: Prochanovs Publizistik wird literaturwissenschaftlich untersucht. Der Autor hat zahlreiche publizistische Texte verfasst - u. a. Zeitungsartikel und Essays. In diesem Beitrag untersuche ich den Zeitungsartikel Stalin - ne bronza, a skorost' sveta 'Stalin ist keine Bronze, er ist die Lichtgeschwindigkeit' ${ }^{6}$, der zunächst 2005 in Zavtra 'Morgen' publiziert wurde und

6 Alle Übersetzungen aus dem Russischen ins Deutsche stammen von mir - M. G. 
im selben Jahr auch im Sammelband Chronika pikirujušego vremeni 'Chronik einer Zeit im Sturzflug' erschien. Dieser Text gilt als repräsentatives Beispiel, weil er die wesentlichen tropologischen und thematischen Merkmale der Publizistik Prochanovs aufweist.

Stalin - ne bronza, a skorost' sveta weist aus literaturwissenschaftlicher Perspektive folgende Merkmale auf: Literarizität (aufgrund der poetischen Sprachfunktion und der Tropen), Selbstreferenzialität und Fiktionalität. Die Tropen enthüllen die Figurativität des Textes und klammern jegliche Wahrheitsansprüche aus, was sich wiederum auf die Lektüre niederschlägt: Es ist nicht relevant, ob die im Text konstruierten ideologischen und politischen Bilder mit der Realität übereinstimmen. Tropen sind Schauplätze der Selbstreferenzialität und der Autodekonstruktion des Textes und manifestieren die performative Gewalt der Sprache, die eine ideologisierte textuelle Welt dort konstruiert, wo Bilder durchgesetzt werden.

Eine dekonstruktivistische Untersuchung der tropologischen Struktur von Stalin - ne bronza, a skorost' sveta bringt jenes doppelte textuelle Verfahren der Konstruktion und (Auto-)Dekostruktion ans Licht, das ich bereits erwähnt habe. Die Autodekonstruktion des Textes hat Auswirkungen auf das Verhältnis zwischen Text und Autor. Stalin - ne bronza, a skorost' sveta wird zu einem automatisierten Verfahren, zu einer Maschine zur Ausschaltung des Autors. Die Hauptfunktion der literarischen Sprache im Text besteht also darin, dass das Verhältnis zwischen dem Verfasser und dem Text zu einem Chiasmus wird: Der Text reflektiert die Autorinstanz ${ }^{7}$ und wirkt auf sie zurück. In diesem Fall kann von einem linearen und unidirektionalen Verhältnis nicht die Rede sein. Die (schon fraglichen), Grenzen` der Publizistik sind somit weit überholt.

Die Verschiebung des wissenschaftlichen Fokus von dem Autor auf den Text soll hier keine Verharmlosung der von Prochanov vertretenen neostalinistischen und neoimperialistischen Ideologie bedeuten. Dass diese ideologische Konstellation eine gewalttätige ist, ist unumstritten. Die symbolische Gewalt der Sprache spielt dabei selbstverständlich eine zentrale Rolle. Prochanovs Romane, Essays und Zeitungsartikel sind Manifestationen des Politischen ${ }^{8}$, in denen die Rhetorizi-

7 Im Dekonstruktivismus ist es natürlich nicht üblich, von Autorinstanz zu sprechen. Da aber Prochanovs Texte sich an der Schnittstelle zwischen Belletristik und Publizistik befinden, erlaube ich mir, auch diese Kategorien miteinzubeziehen.

8 Diesbezüglich mag es hilfreich sein, anhand Chantal Mouffe zwischen political und politics zu unterscheiden: Als political bezeichnet Mouffe „the dimension of antagonism which [is] constitutive of human societies“ (Mouffe 2005: 9) und unter politics versteht sie „the set of practices and institutions through which an order is created, organizing human coexistence in the context of conflictuality provided by the political“ (ebda.). Die Ebene der politics wird in meiner Unter- 
tät und Bildhaftigkeit der Sprache zu politischen Zwecken genutzt wird. Metaphern kristallisieren allgemeine Vorstellungen, Werte und Erwartungen und übertragen sie in Bilder, die ein emotives und persuasives Potenzial haben und komplexe Konzepte verständlicher, anschaulicher machen ${ }^{9}$. Auch in Prochanovs Publizistik lassen Tropen ideologische Werte und politische Positionen zum Ausdruck kommen und werden sehr oft im Rahmen eines aggressiven Sprachgebrauchs eingesetzt.

Die Analyse dieses Beitrags erfolgt aus einer literaturwissenschaftlichen Perspektive. Im Vordergrund steht nicht die Frage danach, welche Rolle Prochanovs Texte in der heutigen russischen Politik spielen, sondern die Frage nach ihrer tropologischen und literarischen Struktur. Der Fokus liegt dabei auf dem selbstreferenziellen und metaliterarischen Charakter der Allegorien, Metaphern und Anthropomorphismen. Die Rhetorik der Tropen (vgl. Speck 1997: 99) enthüllt „das Arbiträre des Zuordnungsverfahrens" ${ }^{10}$ von Stalin - ne bronza, a skorost' sveta und bringt das aporetische Verhältnis zwischen dem Text und seiner Interpretation ans Licht (vgl.: de Man 2003: 98; Speck 1997: 106).

\section{Prochanovs tropologischer Neostalinismus}

In Stalin - ne bronza, a skorost' sveta spricht sich Prochanov für die Rehabilitierung des Stalinismus aus. Der Autor liefert keine objektive Argumentation, sondern arbeitet mit dem figurativen Sprachgebrauch, mit dessen Hilfe er seine Ideologie verbildlicht. Das Publizistische wird somit durch das Tropologische ersetz ${ }^{11}$ : Es sind die Tropen, die im Vordergrund des Textes stehen und Konzepte versprachlichen, die lediglich auf Textebene in einem Spiel mit Signifikaten und Signifikanten funktionieren. Die Tropen verschieben die Sakralisierung der Per-

suchung ausgeblendet; das Politische ist aber zweifelsohne Grundlage der Literatur Prochanovs und spielt eine große Rolle in der thematischen Struktur seiner Texte.

9 Das besondere Paradoxon besteht dabei darin, dass komplexe tropologische Operationen zur Reduzierung der Komplexität der Begriffe eingesetzt werden (vgl. Androshchuk 2014: 72-73 und Weber 1995: 46-47).

10 Müller 1988: 87.

11 Die Bildhaftigkeit des Textes entspricht einem generellen Verfahren bei Prochanov, das zur Medialisierung des Autors führt. Prochanov tritt in seinen Schriften, im Internet sowie in Radiound Fernsehauftritten immer als mediale Instanz auf. Die militärische, orthodoxe und sowjetische Symbolik kommt sowohl in tropologischer Form in den Schriften als auch in Bildform auf dem LiveJournal des Autors zum Tragen (http://ru-prokhanov.livejournal.com/). Auf diesem Blog sind sowohl der bewaffnete Autor (Abb. 1) als auch Bilder der sowjetischen Luftwaffe (Abb. 2) oder orthodoxe und christliche Motive zu sehen (Abb. 3). 
son und der Ära Stalins auf eine figurative Ebene und werden somit selbstreferenziell. Der Selbstverweis ist besonders dort evident, wo Tropen als solche markiert werden: An einigen Stellen im Text heißt es explizit, dass es um allegorische, analogische und metaphorische Bilder geht, wie ich später zeigen werde.

Die tropologische Prägung des Textes kündigt sich schon im Titel an, der aus zwei Analogien - einer negativen und einer affirmativen - besteht. Durch dieses Verfahren ex negativo wird Stalin mit der Lichtgeschwindigkeit identifiziert. Der totalisierende Anspruch der Metapher, den de Man anhand eines Passus aus Marcel Prousts Recherche darlegt (vgl. de Man 2003: 44-47), ist auch in der analogischen Gleichstellung (vgl. Rolf 2010: 77-81) zwischen Stalin und der Lichtgeschwindigkeit zu sehen. Diese tropologische Operation, die nach Hans Georg Coenen als eine ,nicht-triviale Analogie‘ bezeichnet werden kann (vgl. ebda.: 80), identifiziert Stalin mit einer mathematisch messbaren, aber zugleich allmächtig wirkenden Größe und baut dabei eine Herrschaftsstruktur auf, was für die rhetorische Sakralisierung des Politischen üblich ist ${ }^{12}$.

Diese Analogie erscheint wegen der Möglichkeit, im Russischen das Verb ,sein' im Präsens zu elidieren, als besonders kräftig: Die zwei Elemente dürfen ohne ein Verbindungselement gleichgesetzt werden. Ähnlich funktioniert die Analogie „Памятник Сталину - это великая >красная цивилизацияь, подобной которой не знало человечество во времена фараонов, эллинизма, древнего и нового времени“"13, bei der sich mehrere tropologische Transformationen miteinander verflechten. Erstens wird der Begriff pamjatnik ,Denkmal“ im übertragenen Sinne verwendet, und zwar im Sinne von ,Erinnerung‘, was eine figurative semantische Ebene eröffnet. Zweitens wird er mit Andeutungen auf geschichtliche Ereignisse assoziiert - wodurch die konkrete und die figurative Ebene verschmelzen und die sowjetische Geschichte zur impliziten Allegorie der Zivilisation wird. Dadurch wird auch Geschichte textualisiert. Prochanovs Versuch, ein einzig legitimes Metanarrativ mit einer tropologischen und nahezu postmodernen Ästhetik zu etablieren, hat auch einen aporetischen Charakter: Die postmoderne Feststellung der Unmöglichkeit, Geschichte zu erzählen, kollidiert mit dem (im Fall von Prochanov restaurativen) Wunsch, die Erzählung fortzusetzen. Somit erscheint jede Erzählung zugleich als konstruierte, unmögliche aber trotzdem notwendige.

12 Solche rhetorischen Operationen sind auch für das sowjetische Novojaz charakteristisch (vgl. Weiss 1985: 247-325). Aufgrund von Prochanovs sowjetischer Sozialisierung und politischer Einstellung ist das Novojaz fester Bestandteil seiner Texte.

13 „Das Denkmal für Stalin ist die große `rote Zivilisation‘; eine derartige Zivilisation kannte die Menschheit weder zur Pharaonenzeit, noch im Ellenismus, weder in der Antike noch in der Moderne“. Prochanov 2005: 12. 
Die bildhafte Konstruktion der Geschichte wird in einem weiteren Vergleich deutlich: Das Ideologem ,rote Zivilisation“ wird auch mit der präsowjetischen Geschichte in Verbindung gesetzt - diesmal sind es die Figuren von Petr I. (16721725) und Ivan IV. (1530-1584), die diese enorm ausgedehnte Dimension der Zeit kristallisieren und verkörpern, die nicht mehr linear und chronologisch läuft (vgl. ebd.). Auch die zwei Zaren werden als Beispiele zweier Epochen aufgeführt, die trotz ihres schweren und gewalttätigen Charakters zur Ruhm der russischen Geschichte beigetragen hätten. Die Gegenüberstellung von Petr I. und Ivan IV. gerät in Widerspruch mit seinem dichotomischen Denken, da die zwei Zaren die Verkörperung zweier entgegengesetzter Kulturpolitiken in zwei unterschiedlichen Epochen sind. Dieser Widerspruch legt die rhetorische Natur der Argumentation bloß.

Die durch diese Analogien eröffnete Dimension der Zeitlichkeit spielt eine wesentliche Rolle bis zum Ende des Textes. Stalin wird als eine Kraft präsentiert, die jenseits aller Epochen dennoch zugleich Kernfigur der sowjetischen und postsowjetischen Geschichte ist. Somit gerät der Text in eine Aporie, indem er Stalin als jemand Undarstellbaren und Unbegreiflichen vorstellt, aber zugleich versucht, ihn darzustellen und seine ,Größe‘ zu beweisen. Sämtliche Verbildlichungen Stalins kommen ausschließlich in Form von Tropen vor und erweisen sich als Simulakren im Baudrillard'schen Sinne, die nur innerhalb des Textes eine semiotische Valenz haben: Sie verweisen nicht wirklich auf Fakten, obwohl ihre manifeste symbolische Gewalt einen Anspruch auf Realität und Wahrheit enthüllt.

Die Widersprüchlichkeit zwischen diesem Anspruch und der bloß rhetorischen Natur der Bilder, die Stalin darstellen, wird zu einer Aporie in der Kritik des Autors, die sich auf die Errichtung von Denkmälern für Stalin richtet. Nach Prochanov ist der „мистическ[ий] деятел[ь] минувшего века“14 undarstellbar; wenn Stalin aber nicht verbildlicht werden kann, sollte er auch nicht in sprachlichen Bildern dargestellt werden. Der aporetische Charakter dieser Stelle ist auch in der Aussage „Я против постановки памятников“15 evident. Hier taucht die Stimme des Autors auf - allerdings in figurativer Form. Leserinnen und Leser können hier nicht entscheiden, ob sie einen Selbstverweis auf die konkrete Person Aleksandr Prochanov oder ein neues Simulakrum vor Augen haben. Diese Aporie macht auch eine eindeutige Gattungszuordnung unmöglich: Gehört die Verwendung der Ich-Form in diesem Fall zum üblichen publizistischen Stil, oder ist das Ich vielmehr eine rhetorische Figur, die am tropologischen Spiel des Textes partizipiert?

14 „Der mystische Staatsmann des vergangenen Jahrhunderts“. Prochanov 2005: 11-12.

15 „Ich bin gegen die Errichtung von Denkmälern“. Prochanov 2005: 11. 
Die Tropologisierung der Geschichte wird im Text fortgesetzt. Die Entstalinisierung erscheint als leere Allegorie von sich selbst, weil sie in Form von rhetorischen Figurationen versprachlicht wird. Die gesamte Kette von Tropen, die schon auf der ersten Seite in Gang gesetzt wird, dekonstruiert den Text: Sie inszeniert Bilder, die stark mythologisiert und sakralisiert sind und auf einer verschwörerischen Geschichtsvorstellung basieren ${ }^{16}$. Das Bild des Anathems des XX. Parteikongresses (vgl. ebda.: 11) konstituiert den Höhepunkt der Allegorisierung einerseits und enthüllt die semiotischen Prozesse der Sakralisierung des Stalinismus andererseits. Die Kette der Metaphern und Allegorien mündet in das Bild der Kathedrale des seligen Basilius, die wie das Bild des Anathems auf einer Metaebene in doppelter Hinsicht operiert. Die Kathedrale erscheint als Allegorie der Sakralisierung Stalins (durch eine Identifizierung zwischen Stalin und Ivan IV., die deutlicher sowjetischer Prägung ist ${ }^{17}$ ) einerseits und als autodekonstruktive Trope andererseits: Die Kathedrale wird im Text explizit als Bild definiert: „,X]pam Василия Блаженного - образ русской мечты, русского рая“"18. Das Wort obraz ,Bild‘ legt die tropologische Natur dieses Bildes bloß, das als eine Allegorie im klassischen Sinne explizit markiert wird: Chram ,Kathedrale‘ und russkaja mečta ,russischer Traum' werden nicht analogisch gleichgesetzt (wie es im Titel der Fall war), sondern sie werden durch obraz in Verbindung gebracht - und damit ausgerechnet durch das Wort, das das rhetorische Verfahren selbst benennt. An dieser Stelle ist das Spiel mit der Sprache zweifelsohne selbstreferenziell.

Das Wort obraz ist auch aus einem anderen Grund relevant: Es wird mit zwei Genitiven „русской мечты, русского рая“"19 ergänzt, die zum Bild einer mystischen „государственность“20 führen und in den Satz „Чужак не поймет только свой“ ${ }^{\text {‘21 }}$ münden. Dieser Satz expliziert die binäre Logik des Textes, in

16 Dieses Thema kommt in der russischen Gegenwartsliteratur nicht selten vor: Man denke nur an Dmitrij Bykovs $\check{D} D$ (2006) oder an Zahar Prilepins publizistische Texte, die in dem Band Ja prišel iz Rossii 'Aus Russland bin ich gekommen' (2009) gesammelt sind. Dabei ist es wichtig zu bemerken, dass das Motiv der Verschwörung in $\breve{D} D$ mit einer Rehabilitation des Stalinismus nicht verbunden ist, sondern es greift andere Blickrichtungen auf. Aus räumlichen Gründen kann dieser Punkt hier nicht ausführlicher dargelegt werden.

17 Ich beziehe mich auf Sergej Ejzenštejns zweiteiligen Film Ivan Groznyj (1944 und 1958), dessen Realisierung Stalin persönlich in Auftrag gab. Prochanovs Gleichstellung von Stalin und Ivan IV. ist daher eine leere Trope, weil sie sich wiederum auf Stalins eigene Identifizierung mit Ivan bezieht.

18 „Die Basiliuskathedrale ist das Bild des russischen Traums, des russischen Himmelreichs“. Prochanov 2005: 12. Hervorhebung M.G.

19 „Des russischen Traums, des russischen Himmelreichs“. Ebenda.

20 „Staatswesen“. Ebenda.

21 „Der Fremde wird's nicht verstehen - nur der Unsrige“. Ebenda. 
dem Tropen oft in kontrastiven Paaren verwendet werden, um eine Ideologie des ,Unsrigen‘ zu etablieren, die den politischen Manichäismus des Kalten Krieges und die religiöse Dämonisierung der ,Heiden` verbindet.

Diesen doppelten manichäischen Charakter hat auch die Analogie „Победа 45-го года - это вершина мировой истории, мистический пик“22, die ihre totalisierende Macht deutlich ausdrückt: Die ganze Menschheit hätte 1945 den Höhepunkt der ganzen Geschichte erreicht und deshalb hätte eine komplette Fusion von Macht und Volk stattgefunden („власть обнялась с народом“23). Durch ein inverses synekdochisches Verfahren werden die Elemente ,Volk‘ und ,Macht' genannt, die konstruierte Gesamtheiten von kleineren Teilen sind: ,Macht“ steht für die konkreten Machtorgane, wie ,Volk‘ für eine imaginäre Gruppe von Menschen steht. Die Verwendung dieser ,leeren Signifikanten' dient hier zu hegemonialen Zwecken. Insbesondere das Zeichen ,Volk‘ wird zu einem nodal point, das die Pluralität der sozialen und politischen Identitäten nivelliert und eine Äquivalenz durchsetzt mit dem Ziel, eine homogene Gesellschaft zu konstruieren:

\footnotetext{
Any discourse is constituted as an attempt to dominate the field of discursivity, to arrest the flow of differences, to construct a centre. [...] The practice of articulation [...] consists in the construction of nodal points which partially fix meaning; and the partial character of this fixation proceeds from the openness of the social, a result, in its turn, of the constant overflowing of every discourse by the infinitude of the field of discursivity. (Laclau \& Mouffe 2014: 99).
}

Stalin erscheint erneut als die Figur, die diesen tropologischen und hegemonialen Fusionsprozess kristallisiert und vereinigt, indem der sowjetische Leader als die Verkörperung der Macht und der Geschichte präsentiert wird: „И все это соединилось в Вожде, олицетворилось в Сталине“24. Das Bild Stalins wird selber zu einem nodal point und nimmt ferner psychoanalytische Züge: Als otec narodov ,Vater der Völker‘ übernimmt Stalin die Lacansche Funktion eines point de capiton:

Lacan has insisted on [the] partial fixations through his concept of points de capiton, that is, of privileged signifiers that fix the meaning of a signifying chain. This limitation of the productivity of the signifying chain establishes the positions that make predication possible. (Laclau \& Mouffe 2014: 99).

Stalin als Vater und Herrscher der symbolischen Ordnung übernimmt die Spitze einer hegemonialen Herrschaftsstruktur, deren Homogenität auf eine tropologi-

22 „Der Sieg von 1945 ist der Höhepunkt der Weltgeschichte, ein mystischer Gipfel“. Ebenda: 13.

23 „Die Macht hat sich mit dem Volk umarmt“. Ebenda.

24 „Und das alles hat sich in dem Führer vereinigt, hat sich in Stalin verkörpert“. Prochanov 2005: 13. Hervorhebung M. G. 
sche (De-)Konstruktion einerseits und auf die Abgrenzung von dem čužoe ,dem Anderen' angewiesen ist. Die Dämonisierung des Anderen erfolgt hier mittels einer tropologischen Operation, bei der Stalin die Totalität der einzelnen Teile durch die Kristallisierung eines leeren Signifikanten repräsentiert. Diese hegemoniale Operation gewinnt [ihre] Identität erst über die Abgrenzung von einem Außen, über den ,negativistischen 'Weg der Verwerfung eines radikalen Anderen, der damit zum paradoxen ,konstitutiven Außen' avanciert (Reckwitz \& Laclau 2006: 344).

Olicetvorilos' ist ein Anthropomorphismus. Durch die Verwendung dieser Trope werden die totalisierende Macht und der selbstreflexive Charakter der Tropen am stärksten - stärker als bei der Metapher, der Analogie und der Allegorie - expliziert. Wie de Man behauptet hat, ist der Anthropomorphismus

eine Identifizierung auf der Ebene der Substanz. Er [...] nimmt etwas als etwas anderes, das er dann als gegeben behaupten kann. Anthropomorphismen frieren die [...] Kette tropologischer Transformationen [...] auf eine einzige, wesentliche Bedeutung ein. (De Man 2003: 181).

Darüber hinaus wird der tropologische Transformationsprozess an dieser Stelle durch die Verben „соединилось [...], олицетворилось [...], обрело“25 expliziert. Der Anthropomorphismus schließt die Tropenkette über Stalin und stellt den Höhepunkt des sich selbst enthüllenden tropologischen Verfahrens des Textes dar.

\section{Fazit}

Stalin - ne bronza, a skorost' sveta präsentiert eine elaborierte tropologische Struktur, die die Dimension des Publizistischen transzendiert und eine deutliche Selbstreferenzialität und Rhetorizität aufweist. Eine dekonstruktivistische Analyse zeigt, dass diese Rhetorizität nicht als bloße Manipulierung der Sprache zu politischen Zwecken erklärt werden kann. Tropologische Transformationen werden im Text sehr oft markiert, sodass das Verfahren explizit wird und die verwendeten Bilder auf den Rezipienten leer und konstruiert wirken. Die Bildhaftigkeit, die symbolische und totalisierende Macht und die Selbstreferenzialität der Tropen hängen im Fall von Stalin - ne bronza, a skorost' sveta in erster Linie nicht von einem Missbrauch der Rhetorik zu politischen und ideologischen Zwecken ab, sondern von der Grammatik und der Rhetorizität (im Sinne de Mans) der

25 „Vereinigt [...], verkörpert [...], gewonnen“. Prochanov 2005: 13. 
Sprache, die solche Strukturen überhaupt ermöglicht (vgl. de Man 2003: 148). Die Bildhaftigkeit des Textes dekonstruiert nicht nur die tropologische Kette, sondern auch die Autorschaft: Der Text wird zu einem automatisierten Verfahren, das Prochanovs Autorschaft infrage stellt und es unmöglich macht, von einem klassischen Fall von Publizistik zu sprechen. Die Autorinstanz wird zu einer Trope unter anderen Tropen. Die von Prochanov angestrebte Absenz von Ironie wird somit fraglich: Die konsequente Kluft zwischen Signifikaten und Signifikanten, die aus der tropologischen Struktur des Textes resultiert, generiert bei der Lektüre und bei der Interpretation aporetische Irritationen, die nur bei einem dekonstruktivistischen Ansatz ans Licht kommen. Die Dekonstruktion ermöglicht, auch in Verbindung mit Ernesto Laclaus Hegemonietheorie, die Interpretation der literarischen Publizistik als manipulierte Literatur zu überwinden.

\section{Literaturangaben}

Androshchuk, B. 2014. Die Metapher als ideologisches Instrument der Persuasion im deutschsprachigen politischen Diskurs: Eine Untersuchung anhand der Familienpolitik der 16. Legislaturperiode des Deutschen Bundestages. Hamburg: Dr. Kovač.

Averbeck-Lietz, S. 2013. Publizistik. In G. Bentele \& H. Brosius (Hrsgg.), Lexikon Kommunikations- und Medienwissenschaft, 285-286. Wiesbaden: Springer.

Beljakov, S. 2009. Etjud v krasno-koričnevych tonach. Aleksandr Prochanov. In Voprosy literatury 5 (2009). http://magazines.russ.ru/voplit/2009/5/. Letzter Zugriff: 30.07.2015.

Bourdieu, P. \& J. C. Passeron. 1973. Grundlagen einer Theorie der symbolischen Gewalt. Frankfurt a. M.: Suhrkamp.

Bourdieu, P. 1992. Les règles de l'art. Genèse et structure du champ littéraire. Paris: Éd. du Seuil.

Danilkin, L. 2007. Čelovek s jajcom. Žizn'i mnenija Aleksandra Prochanova. Moskva: Ad Marginem.

Erofeeva, M. 2007a. Chudožestvennost’ i publicistika v romane Aleksandra Prochanova «Gospodin Geksogen». In Slovo: Sborniknaučnych rabotstudentovi aspirantov 5 (2007).179-183.

Erofeeva, M. 2007b. Sintez chudožestvennoj literatury i publicistiki v romane A. A. Prochanova «Iduščie v nočì. In Vestnik Kostromskogo gosudarstvennogo universiteta imeni N. A. Nekrasova 2 (2007). 50-52.

Klitsche-Sowitzki, U. 2011. Eurasismus und Neoeurasismus in Russland: Historischer Abriss und Funktionsanalyse des Raumkonzeptes Eurasien. In G. Lehmann-Carli \& Y. Drosihn (Hrsgg.), Russland zwischen Ost und West? Gratwanderungen nationaler Identität, 81-160. Berlin: Frank \& Timme.

Laclau, E. \& C. Mouffe. 2014. Hegemony and Socialist Strategy: Towards a Radical Democratic Politics. London: Verso.

Lipoveckij, M. 2008. Paralogii. Transformacii (post)modernistskogo diskursa v russkoj kul'ture 1920-2000-ch godov. Moskva: NLO.

de Man, P. 2003. Allegorie des Lesens. Frankfurt a. M.: Suhrkamp.

Mey, A. 2004. Russische Schriftsteller und Nationalismus 1986-1995. Bochum: Projektverlag. 
Moebius, S. \& A. Wetterer. 2011. Symbolische Gewalt. In Österreichische Zeitschrift für Soziologie 36 (2011). 1-10.

Mouffe, C. 2005. On the Political. London \& New York: Routledge.

Müller, H. 1988. Kleist, Paul de Man und Deconstruction. In J. Fohrmann \& H. Müller (Hrsgg.), Diskurstheorien und Literaturwissenschaft, 244-257. Frankfurt a. M.: Suhrkamp.

Prochanov, A. 2005. Stalin - ne bronza, a skorost' sveta. In A. Prochanov, Chronika pikirujušego vremeni, 11-13. Ekaterinburg: Ul'tra Kul'tura.

Reckwitz. A. 2006. Ernesto Laclau: Diskurse, Hegemonien, Antagonismen. In S. Moebius \& D. Quadflieg (Hrsgg.), Kultur: Theorien der Gegenwart, 339-349. Wiesbaden: Springer Verlag.

Rich, E. 1995. Aleksandr Prochanov. In South Central Review 12 (1995). 18-27.

Rolf, E. 2010. Metaphertheorien. Typologie, Darstellung, Bibliographie. Berlin: de Gruyter.

Speck, S. 1997. Von Šklovskij zu de Man. Zur Aktualität formalistischer Literaturtheorie. München: Fink.

Weber, F. 1995. Denken in Metaphern. Kognitive Semantik und französische Gefühlsmetaphorik. Frankfurt a. M. \& Berlin: Lang.

Weiss, D. 1985. Was ist neu am "Newspeak"?: Reflexionen zur Sprache der Politik in der Sowjetunion (= Slavistische Linguistik, 1985), 247-325. München: Sagner.

\section{Internetquellen}

http://nbp-rostov.front.ru/. Letzter Zugriff: 29.07.2015.

http://www.zavtra.ru/content/view/2005-04-2011/. Letzter Zugriff: 29.07.2015.

http://ru-prokhanov.livejournal.com/. Letzter Zugriff: 14.06.2016. 